\title{
The BINSYN Program Suite
}

\author{
Albert P. Linnell \\ Department of Astronomy, University of Washington Seattle, WA, USA \\ email: linnell@astro.washington.edu
}

\begin{abstract}
The BINSYN program suite has been ported to a Linux-based operating system. The new program structure is a major revision from the original version and a public version is nearing completion. This paper describes research areas where the program suite is particularly applicabile.
\end{abstract}

\section{Introduction}

BINSYN is a general purpose program package for analysis of binary stars with or without an optically thick accretion disk. It simulates either photometric or spectroscopic data or both. The original version simulated light curves using a black body approximation; the basic design features are in Linnell (1984). A differential corrections capability was added in Linnell (1989). Linnell \& Hubeny (1994) added the capacity to calculate synthetic spectra of binary star systems and Linnell \& Hubeny (1996) extended the simulation to include binary stars with optically thick accretion disks.

Until 2009, the suite ran on a Windows operating system compiled under Microsoft Powerstation Fortran. At that time, the decision was made to convert to a VMWare virtual workstation running Linux Ubuntu. The conversion entailed major restructuring of the program intercommunications and a plan was implemented to develop a publiclyaccessible version. A fortuitous contact with Mr. Paul DeStefano, a local Linux expert, led to adoption of the Git Version Control System as the appropriate vehicle to accomplish the objective. Mr. DeStefano has been instrumental in promoting this objective and he remains a development participant. As of the date of this report, the software is stable with 71 programs and 89,000 lines of code. The programs have been written in FORTRAN 77 and compiled with GNU gfortran. Several included tutorial programs remain to be completed and a Users Guide is in preparation.

\section{Some Program Suite Features}

The primary design approach is to divide the simulation task into a series of consecutive program units linked by scripts. To illustrate this, an initial program called CALPT produces photospheric potentials for the two component stars on the Roche model. A following program, PGA, defines the photospheres with grids of colatitude and longitude contours with assignable angular resolution. Subsequent programs produce plane of the sky projections for one or more orbital positions, determine $T_{\text {eff }}$ values at grid nodes for both components, calculate light intensity values toward the observer at each grid node, and finally sum the intensity values with due allowance for eclipse effects. The $T_{\text {eff }}$ values include allowance for gravity darkening and mutual irradiation and the intensity values are for a list of wavelengths.

Additional program units produce a synthetic spectrum of the binary system including individual spectra of the components and a system spectrum. These units require input spectra, either from the program SYNSPEC (Hubeny, Lanz \& Jeffery 1994) or 
elsewhere, and interpolate to each grid node in $T_{\text {eff }}$ and $\log g$. If the input spectra are obtained from SYNSPEC, the user has the advantage of specifying the wavelength range and resolution. In effect, these latter programs attach a limb-darkened, Doppler-shifted synthetic spectrum to each grid node.

Separate program sequences simulate accretion disk systems and include the simulation of the two stars. The program models an accretion disk as an assembly of concentric annuli. The default model for the accretion disk is Keplerian rotation; a different rotation rate can be specified for individual annuli. The simulations include eclipse effects by either star or the accretion disk and optionally include irradiation of the secondary star by the accretion disk, irradiation of the accretion disk rim by the secondary star, and a hot spot on the rim from impact by the mass transfer stream. The simulation uses an expression for the accretion disk temperature profile more general than the Standard Model but includes the Standard Model as the default model. The basic model uses a black body approximation. An interface to the program TLUSTY (Hubeny 1990) produces annulus models on the TLUSTY approximation and, via the program SYNSPEC (Hubeny, Lanz \& Jeffery 1994), corresponding synthetic spectra. The interface program produces a system synthetic spectrum and separate synthetic spectra of the two stars, the accretion disk face, and the accretion disk rim at each tabular orbital longitude.

The program suite can calculate and store synthetic spectra for multiple orbital longitudes, permitting the generation of light curves by synthetic photometry. Differentials correction optimization of synthetic photometry light curves has been tested, so far for binary systems without accretion disks.

In the Linux version, each program resides in an individual directory immediately below the binsyn root directory. A script in each program directory copies necessary input files from a separate input file directory, runs the program, and copies output files to a separate output file directory. The input files use a template format that makes them convenient for use. Each main program has an output file, among several, that logs the running time and calculated parameter values in an easy-to-read tabular format.

A differentials correction optimization requires partial derivatives of system light with respect to system parameters as a function of orbital longitude. BINSYN uses a numerical analysis procedure including second differences. A set of programs receives specified central reference values of system parameters and upper and lower values for each parameter with symmetric offsets from the central reference value. The corresponding light curves provide first differences and second differences at the tabular orbital longitudes. This program set uses a pre-established file of orbital longitudes that will be used in the simulation. Inclusion of second difference tabulation permits calculation of derivatives anywhere within the bounding interval for a given derivative without recalculation of the first and second differences.

Linnell (1989) discusses the differentials correction solution. The current version of this program module includes explicit choice of what parameters to include, calculation of the covariance matrix, a test for normal distribution of residuals, production of a histogram of residuals, and a criterion for stopping iterations.

\section{Input and output file directories}

There is a general purpose input file directory immediately below binsyn and at the same level as the separate program directories. The motivation for this directory is that it acts as an intermediary between individual program directories and directories for particular binary star systems. Thus, a directory for a particular binary system will contain all the input files needed for a solution. A script in the binary system directory 
copies the complete set of the necessary input files to the general purpose input file directory and starts a run of the solution. The programs in the solution chain successively access the input file directory for the files they need. This procedure permits multiple binary star directories to be set up, each with its appropriate set of parameters. A solution for a given system can be run, suspended, and a run for another system initiated without requiring the user to set the input files of the individual programs. Similarly, there is a general purpose output file directory. On completion of a particular program run, the controlling script in that program directory copies the output files to the general purpose output file directory. At the level of the individual star directory, the master local script controlling the complete solution copies the preset input files to the general purpose input file directory, runs the solution program, and copies the final output files from the general purpose output file directory to the specific binary star directory. The user can remain within the binary directory for the entire solution process and examine the final output files without leaving the directory.

\section{Illustrations of BINSYN applications}

Three papers illustrate the application of BINSYN under diverse circumstances.

Sion et al. (2011) is a study of five AM CVn systems. These are doubly degenerate CV systems with nearly pure He components. We choose EM Ceti for illustration. There is a Hipparcos parallax that sets the scaling factor to superpose a system synthetic spectrum on the available IUE spectrum. We used TLUSTY to calculate an atmospheric model for the WD with a $\mathrm{He} / \mathrm{H}$ number ratio of $10^{4}$. Our study established a need for a narrow accretion disk with a $T_{\text {eff }}$ matching the $40,000 \mathrm{~K}$ WD. It had been suspected that EM Ceti is a direct impact accretor. We calculated the trajectory of the mass transfer stream and find that it would not intersect the WD; the stream does intersect the postulated accretion disk.

Linnell et al. (2010a) is a study of WX LMi, a CV polar whose B light curve is strongly affected by cyclotron radiation. Synthetic B,V,R,I photometry established a distance to the system. A particular problem with this system was synthetic spectra representing a white dwarf, dominating the system spectrum in the UV, combined with a relatively cool $(3300 \mathrm{~K})$ secondary star that dominates the system in the IR.

Linnell et al. (2010b) is a study of the CV RW Sex, a nova-like with an accretion disk which dominates the system spectrum. The study demonstrated the inability of the socalled Standard Model to represent the observed spectrum; the study found a model with a modified radial temperature gradient which fitted the observations to within observational error. It is unknown whether the different model has a more general application to nova-like cataclysmic variables. In this system the fit of the final model to the observed spectra confirmed independent determination of the system distance. BINSYN provides the capacity for distance determination either by calibrated synthetic photometry or by fitting synthetic spectra to calibrated observed spectra.

\section{References}

Hubeny, I. 1990, ApJ, 351, 632

Hubeny, I., Lanz, T., \& Jeffery, C. S. 1994, in Newsletter on Analysis of Astronomical Spectra No. 20, ed. C. S. Jeffery (CCP7; St. Andrews: St. Andrews Univ.), 30

Linnell, A. P. 1984, ApJS, 54, 1

Linnell, A. P. 1989, ApJ, 342, 449

Linnell, A. P. \& Hubeny, I. 1994, ApJ, 434, 738 
Linnell, A. P. \& Hubeny, I. 1996, ApJ, 471, 958

Linnell, A. P., et al., 2010a, ApJ, 713, 1183

Linnell, A. P., et al., 2010b, ApJ, 719, 271

Sion, E. M., et al., 2011, ApJ, submitted

\section{Discussion}

A. PRSA: What is the computational time cost for the light curve synthesis?

A. LinNELL: Using the black body option with the illustration grid resolution, the time per iteration is about a minute. Using the synthetic photometry option, the time per iteration is about one hour. That will improve considerably with projected modification of the grid. 